\title{
Invasive Pre- and Postnatal Genetic Evaluation reduces the Reproductive Risk in the Era of Noninvasive or Minimally Invasive Prenatal Screening Method
}

\author{
${ }^{1}$ Puspal De, ${ }^{2}$ Sudipa Chakravarty, ${ }^{3}$ Amit Chakravarty
}

\begin{abstract}
Introduction: Spontaneous abortion has been reported in 15 to $20 \%$ of all diagnosed pregnancies. The most common cause of spontaneous abortion is chromosomal abnormalities of the embryo. Robertsonian translocation is one of the major chromosomal rearrangements with a prevalence rate of $0.1 \%$ of the general population and $1 \%$ of the infertile population. Robertsonian translocation carriers especially 21,14 are the most common balanced rearrangements among the carrier couples with a history of spontaneous abortion.
\end{abstract}

Materials and methods: Cytogenetic evaluation of both the partners and the child revealed that the child had translocated Down's syndrome and the mother was carrier of balanced Robertsonian translocation of 14q;21q. Amniocentesis of the next pregnancy and detection of chromosomal abnormality in the fetus were done by fluorescence in situ hybridization (FISH) analysis of the amniotic cells with $13,18,21, \mathrm{X}, \mathrm{Y}$ probe mix.

Conclusion: The present case study shows that genetic counseling, cytogenetic evaluation, prenatal diagnosis by amniocentesis, and FISH together help couples with nonhomologous RT and history with syndromic child and repeated abortions to get normal offspring.

Keywords: Balanced chromosomal rearrangement, Chromosomal abnormality, Genetic counselling, Robertsonian translocation, Spontaneous abortion,.

How to cite this article: De $\mathrm{P}$, Chakravarty S, Chakravarty $\mathrm{A}$. Invasive Pre- and Postnatal Genetic Evaluation reduces the Reproductive Risk in the Era of Noninvasive or Minimally Invasive Prenatal Screening Method. Int J Gynecol Endsc 2017; 1(1):35-39.

Source of support: This research received no specific grant from any funding agency in the public, commercial or not-forprofit sectors. All the research work was done by the affiliated institutions' funding.

Conflict of interest: None

\section{INTRODUCTION}

Generally, couples planning for their first pregnancy remain unaware of any reproductive problems. Every

\footnotetext{
${ }^{1}$ Assistant Professor, ${ }^{2}$ Professor, ${ }^{3}$ Director

1,2Department of Genetics, Institute of Genetic Engineering Kolkata, West Bengal, India

${ }^{3}$ Department of Biotechnology, Institute of Genetic Engineering Kolkata, West Bengal, India

Corresponding Author: Puspal De, Assistant Professor Department of Genetics, Institute of Genetic Engineering Kolkata, West Bengal, India, Phone: +919007587067, e-mail: puspal.dey@gmail.com
}

sixth couple experiences difficulties in pregnancy outcomes worldwide. ${ }^{1}$ Around 15 to $20 \%$ of all pregnancies in humans end in spontaneous abortion, and the risk in future pregnancy reaches up to $33 \%$ in patients with recurrent miscarriage. ${ }^{2} \mathrm{So}$, now, the clinician feels that the patients deserve evaluation even after only two recurrent miscarriages, though historically defined recurrent spontaneous abortion is three or more consecutive pregnancy losses before 20 and 22 weeks of gestation. ${ }^{3}$

According to studies, the prevalence of chromosomal aberration is approximately $8 \%$ in cases suffering from reproductive problems, and the frequency rises to as high as $50 \%$ in cases of spontaneous recurrent abortions. ${ }^{4}$ The cause is unknown in many instances, but a parental chromosomal abnormality has been taken as one of the possible causes for recurrent miscarriages within the first 3 months of pregnancy.

Modern cytogenetic, prenatal cytogenetic testing is very common for the detection of chromosomal abnormality in the fetus currently. It helps to determine prenatally whether the fetus has certain hereditary or spontaneous genetic disorders. Some of these noninvasive tests, such as ultrasonography and certain blood tests, are often part of routine prenatal care. These tests are safe and sometimes help to determine whether more invasive prenatal genetic tests (chorionic villus sampling, amniocentesis, and percutaneous umbilical blood sampling) are needed. Usually, these more invasive tests are done when couples have an increased risk of having a cytogenetically unbalanced zygote.

In the present observation, we studied a couple with history of an abnormal child and three repeated spontaneous abortions. Cytogenetic evaluation of both the partners and the child revealed that the child has translocated Down's syndrome and the mother was carrier of balanced Robertsonian translocation of 14q;21q. Amniocentesis of the next pregnancy at 16 weeks was done and fluorescence in situ hybridization (FISH) analysis of the amniotic cells with $13,18,21, X, Y$ probe mix to find out the presence of any chromosomal abnormality in the fetus. The present case study shows that genetic counseling, cytogenetic evaluation, prenatal diagnosis by amniocentesis and FISH together help in the assessment of the couples with 
nonhomologous RT and history with syndromic child and repeated abortions to get normal offspring.

\section{CASE REPORT}

A nonconsanguineous couple, 30-year-old male and a 27-year-old female, with their 5-year-old mentally retarded child, came to our cytogenetic laboratory for the evaluation of genetic disorder of their child. The child had dysmorphic faces with delayed development, such as delayed neck holding and walking. They had a history of three repeated abortions in the past 2 years. The first abortion was 4 months from pregnancy and the second was at 3rd month of pregnancy. The third was a missed abortion from a 2-month pregnancy. There were no such histories of repeated abortions in any other family member. The cytogenetic evaluation of the fetuses after abortion was not done. The biochemical and hormonal profile of the female partner was in the normal range, and the ultrasonography reports did not show any structural abnormality in the internal organs. The semen analysis profile of the male partner was found normal. In the present status, they wanted to know the possibilities of obtaining a normal child in future.

\section{MATERIALS AND METHODS}

\section{Cytogenetics Analysis}

In our laboratory, we first collected $2 \mathrm{~mL}$ of peripheral blood from both partners and the child in heparinized tubes and harvested white blood cells for karyotyping. ${ }^{5,6}$ Twenty-five metaphases were analyzed and the karyotype was interpreted using the Applied Imaging Software (Cytovision 3.92). The chromosomes were identified and classified according to the guidelines by the International System for Human Cytogenetic Nomenclature (ISCN, 1995). The chromosome profile (loss and gain analysis) of the abnormal chromosome was done with the help of the same software.

\section{Prenatal Diagnosis}

In this procedure, a sample of the fluid that surrounds the fetus (amniotic fluid) was removed. Amniocentesis is usually done at 15 weeks of pregnancy or later; in our case, the amniocentesis was done at 16 weeks of gestation. Before the procedure, ultrasonography was done to evaluate the heart of the fetus, confirm the length of the pregnancy, locate the placenta and amniotic fluid, and determine how many fetuses are present. The fluid contains cells that have been shed by the fetus. These cells are grown in a laboratory in amniomax with fetal bovine serum. The harvested cells were selected for FISH. The five-probe mix $(13,18,21, X, Y)$ for interphase nucleus (Vysis aneuvision probe) was used to determine the abnormality in the fetus. ${ }^{7,8}$ Fifty cells were observed and interpreted.

\section{RESULTS}

Chromosomal analysis revealed an abnormality in the female partner with $45, \mathrm{XX}$, robs $(14 ; 21)$ chromosomal constitution. The female karyotype revealed 45 chromosomes with missing chromosomes of 14 and 21, along with an additional chromosome, which did not fit into any group of the chromosomes in the karyotype. The banding pattern of the short and long arms of the additional chromosome was similar to chromosome 14 and 21, thereby indicating the presence of a nonhomologous RT. Thus, karyotype was confirmed as $45, \mathrm{XX}, \mathrm{rob}(14 \mathrm{q} ; 21 \mathrm{q})$ as depicted in Figures 1B and D. Chromosomal analysis of the male partner showed normal 46,XY karyotype. Chromosomal analysis revealed 46,XY,rob(14q;21q) in the child. The karyotype of the child also shows 46 chromosomes with missing chromosome of 14 along with an additional chromosome, whose banding pattern was similar to the nontranslocated chromosome present in his mother as well as chromosome 14 and 21. So, the child has the translocated Down's syndrome (Figs 1A and C).

The FISH result of amniocytes in interphase nuclei shows presence of two green and two orange signals in first hybridization LSI probe and two aqua and two green signals in second hybridization CEP probe. Two green and two orange signals confirm the presence of one homologous pair of chromosome no. 13 and one homologous pair of chromosome no. 21 in first hybridization, and two aqua signals and two green signals confirm the presence of one homologous pair of chromosome no. 18 and one pair of sex chromosome in second hybridization. The test proves the presence of normal numerical count of specified chromosome only (Figs 1E and F).

\section{DISCUSSION}

The potential factors, such as genetic, anatomic, endocrine, infectious, and immunological factor influenced recurrent spontaneous abortions in varying degrees. But, the proper etiology is often unclear. So, it is necessary to consider each of these contributing factors in repeated spontaneous abortions. Cytogenetic analysis of the couple and if possible of the abortions was the preferential area to the clinician for the detection of chromosomal makeup. ${ }^{3}$

Chromosomal anomalies mainly are of two types numerical and structural. Both the anomalies affect approximately $50 \%$ cases of recurrent spontaneous abortions. The numerical anomalies like trisomy, $\mathrm{X}$ chromosome monosomy, and polyploidy, especially triploidy, are 


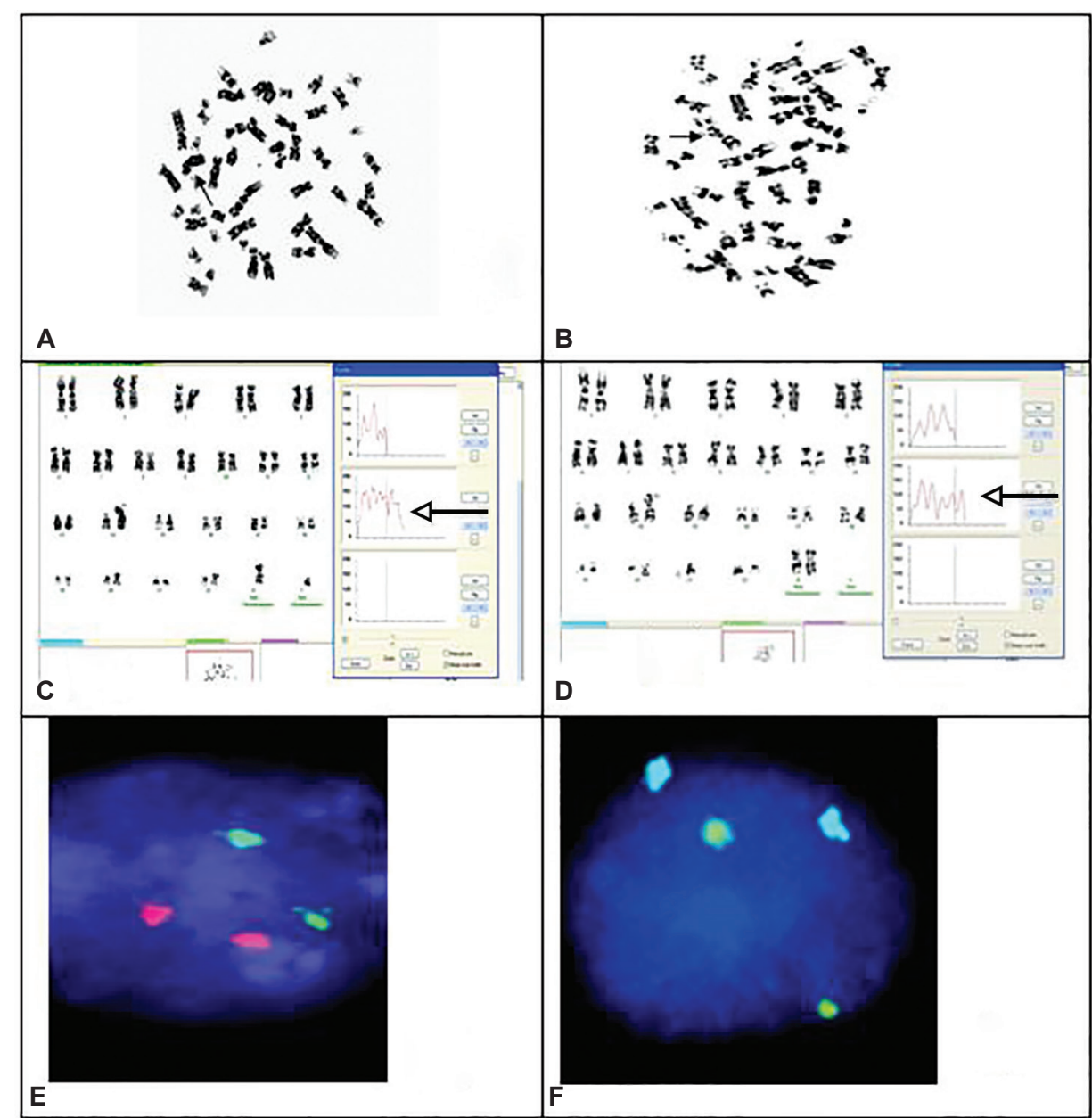

Figs 1A to F: (A) Metaphase spread of affected child. Arrow indicates the translocated $t(14 q, 21 q)$ chromosome; (B) Metaphase spread of the female individual with balanced translocation. Arrow indicates the translocated $\mathrm{t}(14 \mathrm{q}, 21 \mathrm{q})$ chromosome; (C) Karyotype with chromosome profile of affected child. Arrow indicates the gain of materials in chromosome 14q in respect of normal chromosome 14; (D) Karyotype with chromosome profile of the female individual with balanced translocation. Arrow indicates the gain of materials in chromosome $14 q$ in respect of normal chromosome; $(E)$ two green and two orange signals confirms the presence of one homologous pair of chromosome no 13 and one homologous pair of chromosome no 21 in 1 st hybridization; and $(F)$ two aqua signals and two green signals confirms the presence of one homologous pair of chromosome no 18 and one pair of sex chromosome 2 nd hybridization

the main, and they altogether cause $95 \%$ chromosomal abnormalities in recurrent miscarriage cases. ${ }^{9}$ On the contrary, structural anomalies like chromosomal translocations are observed at the highest percentage. Half of the structural chromosomal abnormalities may be inherited from the parent carrying a balanced translocation, which increases the risk of having children with abnormal cytogenetic makeup. ${ }^{10-12}$ At prenatal diagnosis, this risk percentage is considerably higher than the theoretical risk because most cytogenetically abnormal embryos would have failed to establish a pregnancy. So, it is expected that cytogenetic screening of embryos before birth may increase the chance of successful pregnancy. Translocations are of two types - reciprocal and Robertsonian. In reciprocal translocation, the exchange of chromatin blocks between chromosomes is involved with abnormal rearrangements. But the exchange does not affect the gene action. In Robertsonian translocation, two acrocentric chromosomes are fused at their centromeric region and lose their short arm, resulting in a balanced karyotype having only 45 chromosomes including the translocated one. The incidence in newborns is $1.25 / 1,000 .^{13}$ The person with it is called carrier. As carriers are healthy and have normal life span, it is difficult to judge their unusual chromosomal rearrangements. In fact, this translocation can be passed down in families for many generations without discovery. It is noticed only after a baby is born with chromosomal disorder. Only a minor percentage of babies have one parent as carrier, either the translocation is produced de novo at gametogenesis. These de novo unbalanced gametes with Robertsonian translocation have an increased risk of pregnancy loss. According to studies, babies with trisomy 14,15, or 22 are usually miscarried in the first 12 weeks instead of trisomy 
13 or 21 . So, it has been suggested that some carriers are particularly prone to pregnancy loss. ${ }^{14}$

The 1st trimester prenatal screening of Down's syndrome by ultrasound indicates the chance of having a Down's baby, but it does not confirm the same. The ultrasound is called a nuchal translucency test and can be performed when one is between 11 and 14 weeks pregnant. This measures the fluid under the skin at the back of the baby's neck and can be used to determine the risk of having a baby with Down's syndrome. Certain blood tests for screening Down's syndrome are more accurate and are newer options. Presence of human chorionic gonadotropin and pregnancy-associated plasma protein in higher levels in mother's blood also indicates the presence of Down's fetus. There is a lot of dispute about how good these tests are, but they do seem to be pretty accurate. The nuchal cord translucency ultrasound alone probably picks up about $75 \%$ of babies with Down's syndrome, and the blood tests alone, about $60 \%$. If you put the two tests together, the detection rate can be as high as $85 \%$. However, the accuracy of the nuchal translucency ultrasound depends on many factors. So, these kinds of noninvasive or minimally invasive techniques are good for prenatal screening, but when there is increased risk, such as one of the parent is a carrier of balanced translocation, then for further confirmation, certain invasive cytogenetic tests are used for confirmatory diagnostic test. ${ }^{15,16}$

In the present study, the couple under observation showed normal anatomical, endocrinal, and immunological background. The previous blood reports for hormonal and immunological profile of the couple, ultrasonography report of the female, and semen analysis report of the male partner support the above statement. Not only that, they do not have any history of infections in pre-, post-, or during pregnancy. Cytogenetic evaluation revealed normal chromosome profile in the male partner with karyotype of 46,XY, but Robertsonian translocation carrier in female partner with karyotype of $45, X X, \operatorname{rob}(14 q .21 q)$. The cytogenetic evaluation of the child shows translocated Down's syndrome with karyotype of 46,XY,rob(14q:21q). The chromosomal analysis confirmed that the translocation inherited from the carrier mother to child. The couple has the history of three repeated abortions without any chromosome analysis report of aborted fetuses; we could not have any confirmatory idea about these repeated abortions. We think that the translocation derivative chromosomes during segregation may have caused the nonviable pregnancies in these cases.

As one of the parents is normal and other is a balanced translocation carrier, the possibility of getting normal offspring is $50 \%$. But, the present couple has a history of three repeated abortions and a child with translocated Down's syndrome. In this status, it is important to know the chromosomal constituent of their present fetus. For this reason, we performed the chromosomal analysis of the fetus by amniocentasis. The amniocytes are selected for FISH analysis with $13,18,21, \mathrm{X}, \mathrm{Y}$ probe mix and the result showed normal chromosomal constituent without any numerical as well as structural chromosomal abnormality of the fetus, which suggests carrying the pregnancy till birth.

\section{CONCLUSION}

Most couples where one of the partner is a Robertsonian translocation carrier usually do not face any major fertility problems, but in case it appears to be so, it is possible to get help by proper genetic counseling, cytogenetic evaluation, prenatal diagnosis, or by using assisted reproduction. In carrier men, 10 to $15 \%$ of the sperms may be chromosomally unbalanced and in addition, it is thought that the translocation chromosome may block the spermatogenesis. As even men with low sperm counts do produce some sperm, it should still be possible to achieve pregnancy using intracytoplasmic sperm injection. In carrier females, prenatal diognosis or in countries where this is permitted, preimplantation genetic diagnosis may also offer to ensure that implanted embryos are chromosomally balanced. In cases where more than one affected pregnancy or repeated miscarriages are caused by unbalanced chromosomes with or without affected child, the above-mentioned options should be considered.

\section{ACKNOWLEDGMENTS}

Puspal De acknowledges the Institute of Genetic Engineering for funding and affiliation. The authors are also thankful to other laboratory members and other associated persons of IGE for their enthusiastic participation. Authors are especially thankful to Dr Madhumita J Mukhopadhyay for unending cooperation, enthusiasm, and valuable suggestion. Authors also thank Mr Dipak Kundu for computational assistance.

\section{REFERENCES}

1. O'Flynn O'Brien KL, Varghese AC, Agarwal A. The genetic causes of male factor infertility: a review. Fertil Steril 2010 Jan;93(1):1-12.

2. Middeldorp S, Goddijn M. Recurrent miscarriage and thrombophilia. Ned Tijdschr Geneeskd 2006 Jan 28;150(4): 189-193.

3. Dubey S, Chowdhury MR, Prahlad B, Kumar V, Mathur R, Hamilton S, Kabra M, Menon PS, Verma IC. Cytogenetic causes for recurrent spontaneous abortions an experience of 742 couples (1484 cases). Indian J Hum Genet 2005;11(2):94-98.

4. Düzcan F, Atmaca M, Cetin GO, Bagci H. Cytogenetic studies in patients with reproductive failure. Acta Obstet Gynecol Scand 2003 Jan;82(1):53-56. 
5. Moorhead PS, Nowell PC, Mellman WJ, Battips DM, Hungerford DA. Chromosome preparations of leukocytes cultured from human peripheral blood. Exp Cell Res 1960 Sep;20:613-616.

6. Seabright M. A rapid banding technique for human chromosomes. Lancet 1971 Oct 30;2(7731):971-972.

7. Verp MS, Gerbie AB. Amniocentesis for prenatal diagnosis. Clin Obstet Gynecol 1981;24:1007.

8. Shaffer LG, Bui TH. Molecular cytogenetic and rapid aneuploidy detection methods in prenatal diagnosis. Am J Med Genet Semin Med Genet 2007 Feb 15;145C(1): 87-98.

9. Ananthapur V, Avvari S, Tella S, Nallari P, Akka J. A Robertsonian translocation rob $(14 ; 15)$ (q10:q10) in a patient with recurrent abortions: a case report. J Reprod Infertil 2010 Oct;11(3):197-200.

10. Tsui KM, Yu WL, Lo FM, Lam TS. A cytogenetic study of 514 Chinese couples with recurrent spontaneous abortion. Chin Med J (Engl) 1996 Aug;109(8):635-638.
11. Regan L, Rai R. Epidemiology and the medical causes of miscarriage. Baillieres Best Pract Res Clin Obstet Gynaecol 2000 Oct;14(5):839-854.

12. Ogasawara M, Aoki K, Okada S, Suzumori K. Embryonic karyotype of abortuses in relation to the number of previous miscarriages. Fertil Steril 2000 Feb;73(2):300-304.

13. Gardner RJM, Sutherland GR. Chromosome abnormalities and genetic counseling. 2nd ed. Oxford: Oxford University Press; 1996.

14. Scriven PN, Flinter FA, Braude PR, Ogilvie CM. Robertsonian translocations-reproductive risks and indications for preimplantation genetic diagnosis. Hum Reprod 2001 Nov;16(11):2267-2273.

15. Smith-Bindman R, Hosmer W, Feldstein VA, Deeks JJ, Goldberg JD. Second-trimester ultrasound to detect fetuses with Down syndrome. JAMA 2001 Feb 28;285(8):1044-1055.

16. Stewart TL, Malone FD. First trimester screening for aneuploidy: nuchal translucency sonography. Semin Perinatol 1999 Oct;23(5):369-381. 